\title{
ON THE CONDITION
}

OF THF

\section{STOMACH AND INTESTINES IN SCARIATINA.}

BY

SAMUEL FENWICK, M.D., LATE LECTURER ON PATHOLOGICAL ANATOMY AT THE NEWCASTLE-UPON-TYNE OOLLEGE OF MEDICINE IN CONNECTION WITH THE UNIVFRSITY OF DURHAM.

Received April 22nd.-Read June 28th, 1864.

IN the following paper I shall attempt to show, that inflammation of the cesophagus, stomach, and intestines, usually accompanies scarlatina; that, desquamation of the epithelium of these parts takes place; that, notwithstanding the anatomical changes in the stomach, the formation of pepsine is not prevented; and that, in this disorder, the condition of the mucous membrane is similar to the condition of the skin.

I.-That inflammation of the cesophagus, stomach, and intestines usually accompanies scarlatina.

Observation 1.-A child two years old was suddenly attacked by vomiting and diarrhoa. After a few hours a slight scarlet rash appeared on the body; five hours from voL. XIVII. 
the commencement of the illness convulsions came on, and the child died fourteen hours afterwards.

Post-mortem examination.-The mucous membrane of the stomach was in one part much congested, but did not present to the naked eye any other appearance of disease. Its tubes could be separated with greater ease than usual. When examined by the microscope they appeared large and much distended at their closed ends, only a few scattered cells being visible in them, and their basement membrane seemed everywhere lined by a false membrane (see Plate V). After the stomach had been soaked in spirit for six months a considerable change took place. The tubes then seemed filled with cells and with granular matters agglutinated into masses; but the cells were very much less numerous than in the natural condition. Each cell seemed covered with a thin membrane, which could be seen to pass from one to another. The villi of the duodenum were stripped of epithelium, and, although there was no trace of food in the intestines, the villi were distended with oily and granular matters. The lacteals were empty.

Observation 2.-The stomach of a female child, aged twelve years, who had died of scarlatina, was sent me for examination. The tubes were greatly distended and easily separated from each other. In many places they were stripped of their cells, and contained only granular matters, but in other parts there were large and very thin cells in the tubes: The connection between the tubes and their contents was much less than usual, for when sections were made obliquely the tubes were generally found empty. When the cells were removed from the tubes they appeared fringed with pieces of fine membrane.

Observation 3.-The stomach of a female child who had died of scarlatina at the age of two years was sent to me for examination. The tubes were easily separated from each other, and greatly distended by granular and fatty matters. There were but few gastric cells visible, and in 
other respects the appearances were the same as in the preceding case.

Observation 4.-A boy died of scarlatina in the Middlesex Hospital.

Post-mortem examination.-The pharynx and cesophagus were red, and the epithelium was absent. The stomach was covered by a thick tenacious mucus, and when this was removed the mucous membrane was found greatly congested, and in many places presented a blood-stained surface. The intestines were in a similar condition. Peyer's patches were large and congested, and the glands of the mesentery enlarged and of a deep-red colour, as in typhoid fever. The tubes of the stomach were amazingly distended, and their closed ends curved and distorted. After being soaked in alcohol there were spaces left between the basement membrane and their contents. The basement membrane seemed much thickened, and the tubes were gorged with a confused mass of cells and granular matters. Scattered everywhere in apposition with the inner surface of the tubes were small round cells $\frac{1}{4000}$ part of an inch in diameter. There were also a few larger cells $\frac{1}{1100}$ of an inch, very thin and flat, and agglutinated to the rest of the contents. In other specimens no large cells were to be discovered, and in another part the tubes themselves were found united together, the intertubular spaces being loaded with cells and nuclei. The intestinal villi were also loaded with fatty and granular matters, although the lacteals were not distended.

Observation 5.-A girl aged fourteen was admitted into the Middlesex Hospital. She had been affected the night before with sore throat, followed by a slight rash upon the skin. She walked to the hospital in the morning, but was attacked by diarrhœa, and died the same evening.

Post-mortem examination.-The cesophagus immediately above the stomach was very red. The stomach was much injected in the cardiac region, and for some distance from it. There were two or three ulcerations in the solitary glands 
of the intestines. The muscular coats and the subtubular and intertubular spaces of the stomach, were in many parts infiltrated with blood. The tubes were distended and the edges of the cells ill-defined, from being overlaid with granular matters. In the pyloric region were deposits of nuclei encroaching on the tubes; these deposits, no doubt, were the remains of some former attack of inflammation of this part.

Observation 6.-A youth æt. 17 died of scarlatina at the Middlesex Hospital, after a few days' illness.

Post-mortem examination.-The stomach was congested at the pyloric region, but there was no unusual redness in other parts of the organ. In the cardiac and middle regions the tubes were distended with round or oval nucleated cells, about $\frac{1}{4000}$ of an inch in diameter, intermixed with granules and a few gastric cells, some of these being granular and larger than usual. Between and below the tubes were numerous cells and nuclei. In the pyloric region the tubes were greatly distended, and masses of granules and small epithelial cells projected from their free ends. They did not appear to contain any normal gastric cells.

Observation 7.-A man was admitted into the Middlesex Hospital, and died a few days afterwards from scarlatina.

Post-mortem examination.-The cardiac region of the stomach was greatly congested, the mucous membrane being spotted all over with blood. The mucous membrane of the intestines was intensely red. The gastric tubes were very much distended with granular matters and small nucleated cells about $\frac{1}{4000}$ part of an inch in diameter, but no normal gastric cells could be discovered. Between some of the tubes there were also elongated nuclei and a few small cells. The mucous membrane of the duodenum and ileum was everywhere loaded with small cells and granular matters. The villi were very erect, exceedingly large, and deeply coloured by blood, which seemed to have been extravasated 
into their texture, especially at their free ends. They were loaded with small cells and granular matters. The tubes of Lieberkühn were distended with cells and granules. The pancreas was loaded with small cells and granular matters.

Although in all the above cases there was redness of the stomach after death, the amount of disease actually existing in the mucous membrane could not have been suspected had it not been for the microscopic examination. In the next cases, however, the morbid appearances were sufficient to show the nature of the complaint, without the necessity of a more minute investigation.

Observation 8.-In 'Beale's Archives' a case of scarlatina is reported, in which the patient vomited up with some bile " a piece of the epithelial coat of the stomach, about the size of the palm of the hand." At the post-mortem examination "the mucous membrane of the stomach and duodenum was much congested, but there was no extravasation of blood in any part. The muscular coat towards the pyloric orifice was firmly contracted, and the mucous membrane thrown into rugæ, but the cardiac portion was relaxed and the coats in this region seemed very thin."

Observation 9.- The stomach of a child who had died of scarlatina was kindly sent to me by Dr. B. W. Richardson. The stomach and duodenum were intensely inflamed; indeed, excepting the greater brightness of the colour, the mucous membrane presented more the appearance of a case of catarrh arising from heart disease than that of scarlatina. From the fact of the stomach having been preserved in ammonia, I was unable to make any microscopic examination.

Observation 10.-The small intestine of a child who died of scarlatina is preserved in the museum of the Middlesex Hospital, and there are numerous ulcerations which seem to have arisen in the solitary glands. 
In all the above cases death took place within a few days after the attack of the fever, and, in the first, vomiting and diarrhœa preceded the rash. In Dr. Beale's case the vomiting and sore throat are mentioned as occurring on the same day as the rash, but I have generally observed symptoms of gastric disorder before the soreness of the throat has been complained of. It is, therefore, probable that the inflammation of the digestive tube takes place before or at the same time as that of the throat, and is, therefore, prior to the eruption.

The next cases not only strengthen the general conclusion as to the existence of inflammation of the stomach and intestines in scarlatina, but also show the changes which the mucous membrane undergoes in the later stages of the illness.

Observation 11. - A female child, æt. 9; was admitted into the Middlesex Hospital. Two or three weeks previously she had been affected with scarlatina. She died of diseased kidneys, with fluid in the chest. The mucous membrane of the stomach was not injected, but was covered by a thick gelatinous mucus. The tubes were easily separated from each other, and were not thickened. At their closed ends the gastric cells were obscured by a large amount of granular matters, but towards their free extremities the cells were plainly seen, about $\frac{1}{1200}$ of an inch in diameter, round and nucleated, but very much fewer in number than in the natural state.

Observation 12.-A child two years of age passed through the first stage of scarlatina, but died a fortnight afterwards.

Post-mortem examination.-The mucous membrane of the stomach was of a pale colour, and seemed thin and wasted, but in no part did it present any appearance of post-mortem solution. The tubes of the stomach were smaller than usual, and did not show the distended appearance observed in many other cases when death took place 
earlier in the disease. They were lined by a membrane, but many normal cells were visible. After long maceration in spirit the tubes assumed a more natural appearance, and a larger number of cells became visible, although their outlines remained indistinct. In some sections the bloodvessels situated between the tubes were evidently enlarged, but this was by no means general.

Observation 13.-A man twenty years of age was attacked by scarlatina. He was admitted into the Middlesex Hospital, suffering from dropsy, and died about three weeks after the commencement of the fever.

Post-mortem examination.-The stomach was covered by a thick gelatinous mucus. It was not much congested, although the blood-vessels seemed large. At the commencement of the pyloric region the mucous membrane was thick, red, and softer than elsewhere. The tubes seemed loaded with granular matter, but also contained cells, although there were much fewer than usual. There was less than the usual adhesion between the tubes and their contents, for the cutting of the tubes obliquely generally caused them to appear empty.

Observation 14.-A boy was admitted into the Middlesex Hospital. When first attacked, he had suffered from repeated vomitings, but neither sore throat nor eruption on the skin had been observed. He died in two or three weeks after the commencement of his illness.

Post-mortem examination.-The back of the tongue and arches of the palate were stripped of epithelium, but there was not desquamation of the cuticle. In some parts the tubes of the stomach were greatly distended with granular matters, in others the cells were apparent, but seemed matted together and their edges obscured. In other places the tubes themselves were united together, and the spaces below them were loaded with nuclei and small cells about $\frac{1}{5000}$ of an inch in diameter. 
Observation 15.-A man was admitted into the Middlesex Hospital, suffering from scarlatina, which was followed by peritonitis, bloody and albuminous urine, and constant vomiting. Death took place about three weeks after the invasion of the disease.

Post-mortem examination.-The stomach was covered by a thick tenacious mucus, and the mucous membrane was in some parts very dark in colour, almost black; in other parts red and greatly congested, and looked as if blood had been sprinkled upon it; in the middle region it was pale. The tubes were easily separated from each other, and greatly distended by granular and fatty matters. At the closed ends of the tubes the cells were scarcely visible, but were more readily seen when examined towards their free ends. The cells seemed cemented together and covered with fatty granules, and their edges were very indistinct. ${ }^{1}$

The first effects of the scarlatina poison upon the stomach seem to be to congest the blood-vessels, and to strip the epithelium from the tubes and the surface of the organ, at the same time that the tissues are softened. The tubes are greatly distended by granular and fatty matters, or by cells intermixed with granules, whilst in other cases they. are lined by a newly formed membrane. Sometimes no normal cells can be distinguished; in other cases they are present, but are scattered irregularly. After the second or third week the tubes are found less distended, their closed ends are still loaded with granular matters, which greatly obscure the gastric cells, but these become more evident towards

1 The following case has lately come under my notice. A girl, æt. 17, was attacked by scarlatina, and died upon the tenth day. The tubes of the stomach were very much distended by granular matter, and also contained cells which were larger and more granular than natural.

In about two feet of the small intestines, which I had the opportunity of examining, the mucous membrane was covered with a thick tenacious mucus, and only fragments of villi could be discovered, the greater part being quite bare of them. Under the microscope it presented the appearance of a sieve, being everywhere perforated by the enlarged and thickened openings of Lieberkühn's follicles. (See Plate VI, fig. 1.) 
the free surface of the mucous membrane. The cells at this period are sometimes very large, sometimes loaded with fat or coated with granules, and seem still to have but little adhesion to the basement membrane, as they readily separate from the tubes, but adhere closely to each other.

The effects of inflammation upon the intestine seem, in slighter cases, to consist in the effusion of granular and fatty matters into the mucous membrane, but in more severe cases the tubes of Lieberkühn are choked with epithelial cells, whilst extravasation of blood takes place in the villi; and these, with the rest of the mucous membrane, are loaded with small cells and granules. In one case the villi were completely destroyed, and the tubes of Lieberkühn were greatly enlarged and distended by cells and granular matter. In some instances in which the pancreas has been examined appearances of inflammatory action have been remarked.

Tubular inflammation of the stomach is, however, a common disease; and the suspicion may be naturally excited that the appearances above described are nothing more than what might be observed in any other acute disorder.

Out of 150 cases in which I have carefully examined the stomach with the microscope, I have not remarked this condition to be so invariably connected with any other complaint, whilst the coexistence of inflammation of the osophagus, stomach, and intestines, with a similar affection of the throat and skin, clearly points to a general cause.

In addition to this, the appearances presented by the stomach-tubes in scarlatina, when examined in the first stage, often differ considerably from those of ordinary tubular gastritis. The tubes are much more enlarged, especially at their closed ends, and are evenly distended, instead of presenting an uneven outline.' The basement membrane is not so generally thickened, and, in place of being cemented together into little lumps, the gastric cells

1 It is well to state, perhaps, that the most characteristic appearances are met with in young subjects, who have succumbed in the earliest stages of the fever. 
have either disappeared or are quite obscured by newly formed membrane or by fatty and granular matters. The differences between this form and gastritis as it ordinarily occurs, may be observed by comparing Plate V with Plate VI, fig. 2, which represents a section of the stomach taken from a child who died of convulsions on the third day after being attacked by measles. In this figure the normal gastric cells are plainly visible in the tubes, whilst between and below the tubes are numerous nuclei resulting from the inflammation.

As the above sixteen are all the post-mortem examinations I have been able to obtain of persons who have died of scarlatina; and as in all of them the mucous membrane of the digestive tube was more or less inflamed, I think it probable that this condition is a general accompaniment of the fever. As, however, the inflammation varied greatly in degree in different persons, and also in different organs in the same individual, it will, I think, be found that the intensity with which the mucous membranes are attacked varies according to the type of the epidemic and the age and constitutional peculiarities of the person suffering from the disease. The severity of the affection of the stomach and intestines is not necessarily in proportion to that of the skin and throat, for in some of the above cases little redness was observed in the latter when the former were intensely inflamed.

\section{II.-That desquamation of the epithelium of the stomach and intestines takes place in scarlatina.}

It is more difficult to prove that after scarlatina desquamation takes place in the digestive tubes as it does on the skin, for in the majority of cases vomiting only occurs at the outset of the disorder, and I have not had the opportunity of examining the matters ejected from the stomach in this stage of the disease. In Case No. 15 I found casts of the tubes in the fluid vomited, and from this I anticipated the appearances presented on post-mortem examination. 
The chief reason upon which I ground the opinion that desquamation of the epithelium is of common occurrence is derived from the microscopic examination of the contents of the stomachs of those who have died of the fever, and the following are examples of the forms of casts of the gastric tubes as they appear under these circumstances.

In Case 1 there were a few ounces of a brown-coloured turbid fluid in the stomach, which had an alkaline reaction. The thicker portion of the contents, when placed under the microscope, was found to consist of pieces of fine membrane, of cells, granules, and shreds of membrane. The pieces of membrane were everywhere dotted over with granules, some of which were of fat. They varied very much in size, some of them were $\frac{1}{500}$ part of an inch by $\frac{1}{380}$ of an inch, and in many cases a few cells were. attached to their surfaces. The cells, of which the contents mainly consisted, were very similar to those found in a healthy stomach. They varied in size from $\frac{1}{1200}$ to $\frac{1}{2200}$ of an inch, but were more generally about $\frac{1}{1500}$ of an inch in diameter; they were oval or circular in form, and flattened at the sides; they generally contained a nucleus and numerous granules, and were usually attached to fine shreds of membrane. After the addition of acetic acid the membrane became very transparent. (See Plate VII, fig. 1.)

In Case 12 the stomach contained a small quantity of turbid fluid, like gruel. When examined by the microscope it presented numerous pieces of membrane covered with cells, and separate cells, with ragged pieces of membrane hanging to them. The pieces of membrane were of different kinds; some thin, and presenting no appearance of structure, but covered with granules, and with a few nuclei adhering to them, whilst others seemed to consist wholly of cells bound together. In some cases it appeared as if the cells had been stripped from the tubes in a mass, the upper, wider, and flatter part being covered with conical, and the lower, longer, narrower, and more tubular portion being composed of round or oval, cells. The length of these casts varied greatly, and the width was from $\frac{1}{60 \overline{0}}$ to $\frac{1}{100 \overline{0}}$ part of an 
inch; but when their folds were flattened out by the compressor they were much broader. The free cells were like those of a healthy stomach, and varied in size from $\frac{1}{1000}$ to $\frac{1}{2000}$ part of an inch, but usually were $\frac{1}{1600}$ of an inch in diameter. Very few of them had a clear edge, but seemed attached to ragged pieces of membrane, and thus two or three cells were often joined together. (See Plate VII, fig. 1.)

In Case 5 the contents were found to consist chiefly of masses of conical epithelium stripped from the mucous membrane in large flakes. The epithelial cells were overlaid with fine granular matter. There were also numerous casts of the tubes, composed of fine membrane.

But the question arises, whether we can trust to such appearances in an organ so subject as the stomach to postmortem changes. In order to ascertain this, I examined microscopically the contents of the stomachs of forty-five bodies inspected at the Middlesex Hospital, marking in each the condition of the mucous membrane. In one instance only were there any fibrinous casts like those above described, and it occurred in a case of acute gastritis. In eighteen cases there were only separate cells, chiefly of the columnar form; or if there were casts there were very few, and in none of them was there any inflammatory action of the mucous membrane. In eight cases casts of the upper parts of the tubes were plentiful, composed only of healthy conical cells; and in all these the mucous membrane was in a natural condition. In eighteen there were either plugs formed of cells and granules from the secreting parts of the tubes, or the casts of conical cells were overlaid with granular matter, and the stomachs were more or less inflamed. But although $I$ have only found casts of the tubes in one case of scarlatina during life, $I$ have met with them in other diseases in which inflammation of the stomach was present, of which the following are examples.

Observation 16. - A man about 55 years of age had for some years suffered from symptoms attributed to disease 
of the kidney. A fortnight before his death he was attacked with constant vomiting, together with a scalding pain at the epigastrium and between the shoulders, increased by every kind of food. The vomited matters contained fibrinous casts of the stomach-tubes, and latterly, blood.

Post-mortem examination.-Both kidneys were diseased (morbus Brightii), and the stomach was much congested and covered with a thick layer of mucus. When examined by the microscope the tubes were found loaded with granular matter, and their cells cemented together, and with difficulty distinguished.

Observation 17.-A man consulted me suffering from violent pain at the epigastrum after eating, and only relieved by vomiting. The matters vomited early in the morning contained torulæ and numerous casts of the gastric tubes, composed of mucus, covered by small cells. Some I measured were $\frac{1}{33}$ of an inch in length, and $\frac{1}{363}$ of an inch at their closed ends; but towards their free extremities they were only $\frac{1}{571}$ of an inch in diameter. I have also met with tube-casts in cases supposed to be bilious attacks, and in cases of persons suffering from inflammatory dyspepsia.

If, then, we can discover casts of the tubes during life in cases of gastritis, and if, in scarlatina, this condition exists, and casts have been found in the stomach after death, there is, I think, very great probability that desquamation of the epithelium does take place in this organ, as it does on the skin and the kidneys.

The appearances of the stomach-tubes in the foregoing cases of scarlatina corroborate this opinion. In all of them there was a deficiency of the ordinary gastric cells, which were replaced either by small cells or granular matter; and in many instances the contents of the tubes seemed to separate with unusual ease from the basement membrane. 
III.-That, notwithstanding the anatomical changes in the mucous membrane of the stomach, the formation of pepsine is not prevented.

The following experiments prove that the characteristic secretion of the stomach is not destroyed by the injury inflicted upon it by scarlatina.

Two drachms of the mucous membrane were beaten up with one ounce of distilled water and filtered, after standing four hours in a cool place. To this acid was then added, and the albumen was digested in the mixture.

Ten grains of albumen were digested at a temperature of $90^{\circ}$ for twelve hours in an infusion of the mucous membrane taken from Case 7, to which three per cent. of bydrochloric acid had been previously added. Seven and a half grains remained, but in a very pulpy state. A similar experiment was performed with mucous membrane from Case 6, and six and a half grains were left, but very soft and pulpy. Both of these cases had died within the first week of illness, but in a third, who died of congestion of the kidney in the third week, a similar experiment showed a loss of five grains. The average loss was, therefore, three and two third grains. Of eleven males who died of various diseases at the same hospital, similar experiments gave an average loss of four grains, so that little diminution in the amount of pepsine had been produced by the scarlatina. In contrast to this are the results of similar experiments upon four cases who died of typhoid fever. In two the albumen had gained three grains of weight by imbibition, and was not at all softened; in two others it was softened, and in one it had lost balf a grain, in the other a grain and a half. But the activity of the digestion must depend, not only upon the relative amount of pepsine, but also upon the bulk of the mucous membrane. The average weight of the mucous membrane of the stomach of ten males; dying of various diseases, $I$ found to be eighteen drachms; that of the two 
recent cases of scarlatina was eighteen drachms and sixteen drachms ; ${ }^{1}$ whilst it only amounted to fifteen drachms in one who died in the third week. But the loss of substance in typhoid fever appears much greater, as, in three cases I weighed, the mucous membrane only weighed eleven drachms in each.

I am not disposed to attach much importance to the results of the foregoing experiments, as showing the actual amount of pepsine in each case, inasmuch as I had not the means of examining the stomachs under exactly similar conditions, as to the date after death and the nature of the contents; neither would I lay much stress upon the deficiency of pepsine in the cases of typhoid fever as a characteristic of that disease, since $I$ am unacquainted with the state of the patients during life; but as in these three cases of scarlatina the mucous membrane digested an amount of albumen equal to that usually dissolved in persons dying from other diseases, and in most of whom the organ was healthy as to its structure, it is certain that the characteristic secretion of the stomachs had not been destroyed by the inflammatory action.

IV. - That the condition of the skin is similar to the condition of the mucous membrane of the stomach in scarlatina.

I have only examined the skin in three cases of scarlatina. In the first, death took place after a few days' illness, and the only morbid appearance in the cutis was an occasional slight extravasation of blood in the neighbourhood of the sudoriferous ducts. The rete mucosum was greatly thickened; and numerous round cells with large nuclei were everywhere visible, intermixed with the natural cells. The basement membrane of the sweat-glands was thickened, and the epithelial lining so much increased that in most cases it obstructed their channels. In some of the sweat-glands the

1 This was in a boy aged seventeen years. 
coils of which they were composed were loaded with coagulated blood, and were greatly and irregularly distended. (See Plate VII, fig. 2.)

In the other recent case the appearances were similar, excepting that the external layers of the cuticle were stained with blood in minute patches, and the sweat-ducts were also reddened; but there was no extravasation of blood, either in the glands or cutis. In some of the ducts the epithelium was detached from the basement membrane.

In the case of a man.who died in the third week the sudoriferous tubes were still choked up, but in the glands the epithelium seemed in many places to be detached, the basement membrane being bare, or covered only by ragged particles. The cutis was in a natural condition.

It will be remembered that the morbid condition in these specimens is similar to what has been before described as existing in the mucous membrane of the stomach, viz., an increase in the epithelium, with but little affection of the other structures; but we know that in scarlet fever the functions of both . stomach and skin are greatly impaired; and as it has been found that the amount of pepsine is not lessened, may we not attribute the anorexia and the diminution of perspiration rather to the obstructed condition of the glands of these organs than to a loss of secreting power in the cells of their epithelium?

I have, in accordance with custom, described the appearances of the skin and mucous membranes as the result of inflammation; but the following considerations suggest the idea that the term, when so used, is perhaps misapplied.

We find in scarlatina that in each part the morbid condition is mostly confined, in the first instance, to the basement membrane, and consists in the formation of layers of new cells, which in the skin are transformed into cuticle of natural appearance, and in the stomach contain pepsine.

If future researches should prove that a similar condition occurs in the kidney and other organs, we shall have to look upon the structural changes produced as resulting from 
increased physiological action, rather than from inflammatory action, and that the primary effect of the scarlatina poison is suddenly and violently to stimulate the natural cell growths of the various secreting organs; for though it is true that in scarlatina various internal parts are affected by inflammatory action, daily experience shows us that a sudden increase of the normal functions of an organ is apt to produce structural disease of the organ itself.

I would, in conclusion, beg to express my best thanks to Drs. Stewart, Goodfellow, and Thompson, the physicians of the Middlesex Hospital, for their liberality in permitting me to make use of their cases for the illustration of this paper. 


\section{DESCRIPTION OF PLATES V, VI, AND VII.}

\section{Plate V.}

The mucous membrane of the stomach in scarlatina.

$a$. The tubes, greatly distended.

b. A few cells still remaining.

c. Intertubular spaces, free from nuclei or cells.

d. Muscular coat.

\section{Plate VI.}

Fig. 1.-View of the surface of the small intestine in a case of scarlatina.

a. Openings of the follicles of Lieberkühn, much enlarged and thickened. No remains of villi in this specimen.

"2.-Mucous membrane of the stomach in measles.

a. Tubes, containing cells.

b. Gastric cells, cemented together.

c. Nuclei and cells in the spaces between the tubes.

$d$. Nuclei and cells below the tubes. The cells between and below the tubes are not sufficiently numerous in this figure.

\section{Plate VII.}

1) 1.-Tube-casts and cells from the stomach in scarlatina.

a. Tube-casts.

b. Separate cells.

" 2.-Hæmorrhage into a sweat-gland, in a recent case of scarlatina.

The section made in a slanting direction.

$a$. Sections of coiled tube of which the gland is composed.

$b$. A tube greatly distended and altered in shape.

c. The opening of a tube. The opening irregular in shape. 


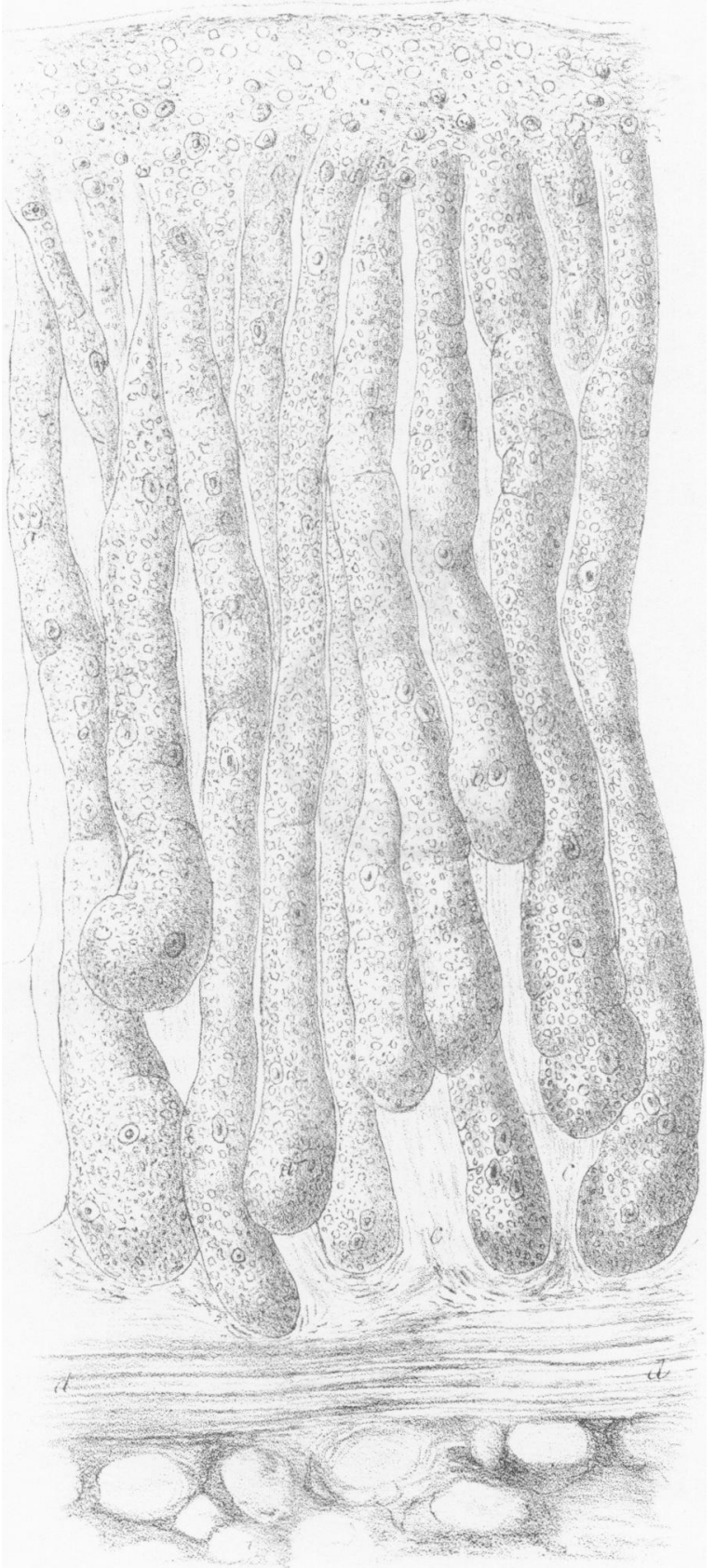




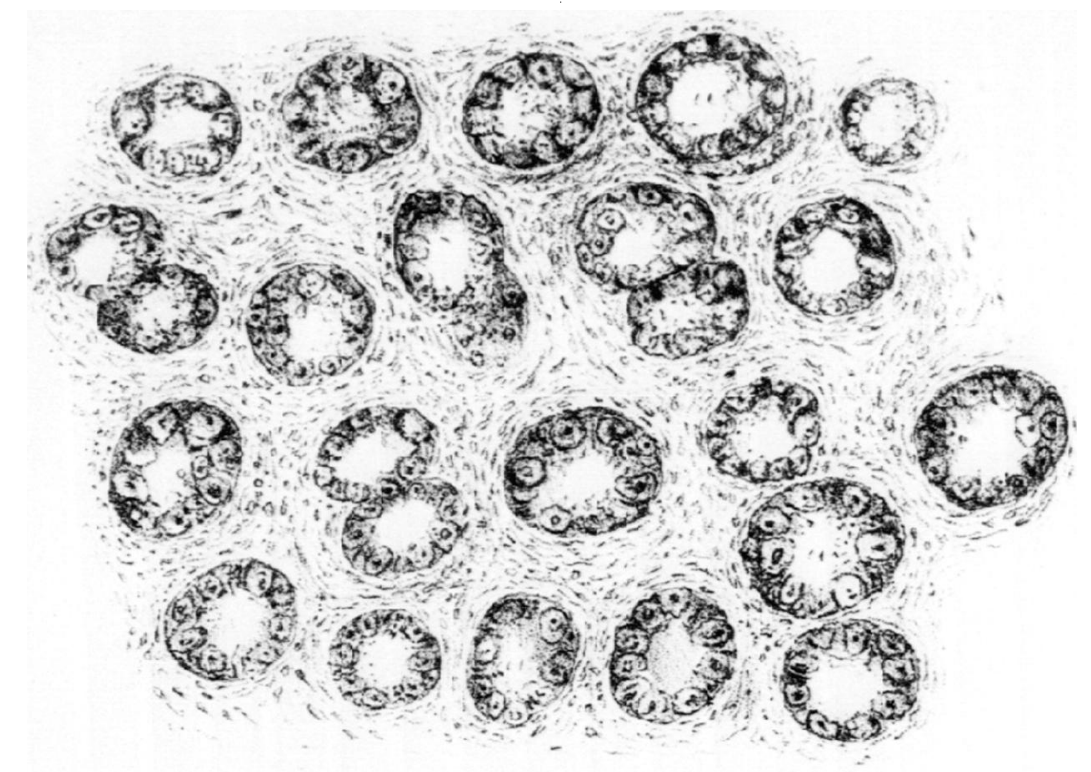

Fig. 2.

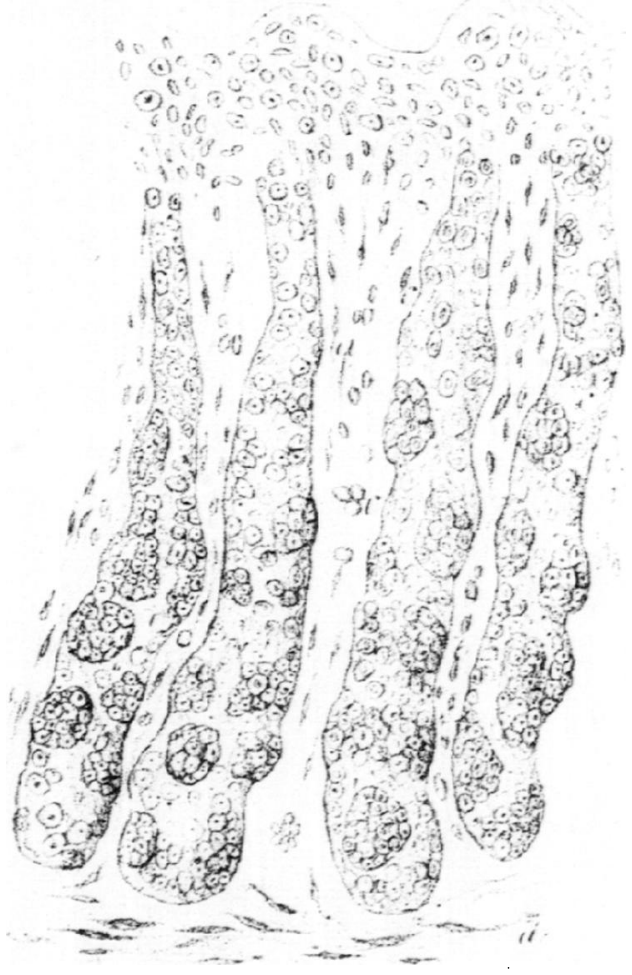

Tuffen West del. G H.F.

W.West imp. 


\section{Fig 1.}

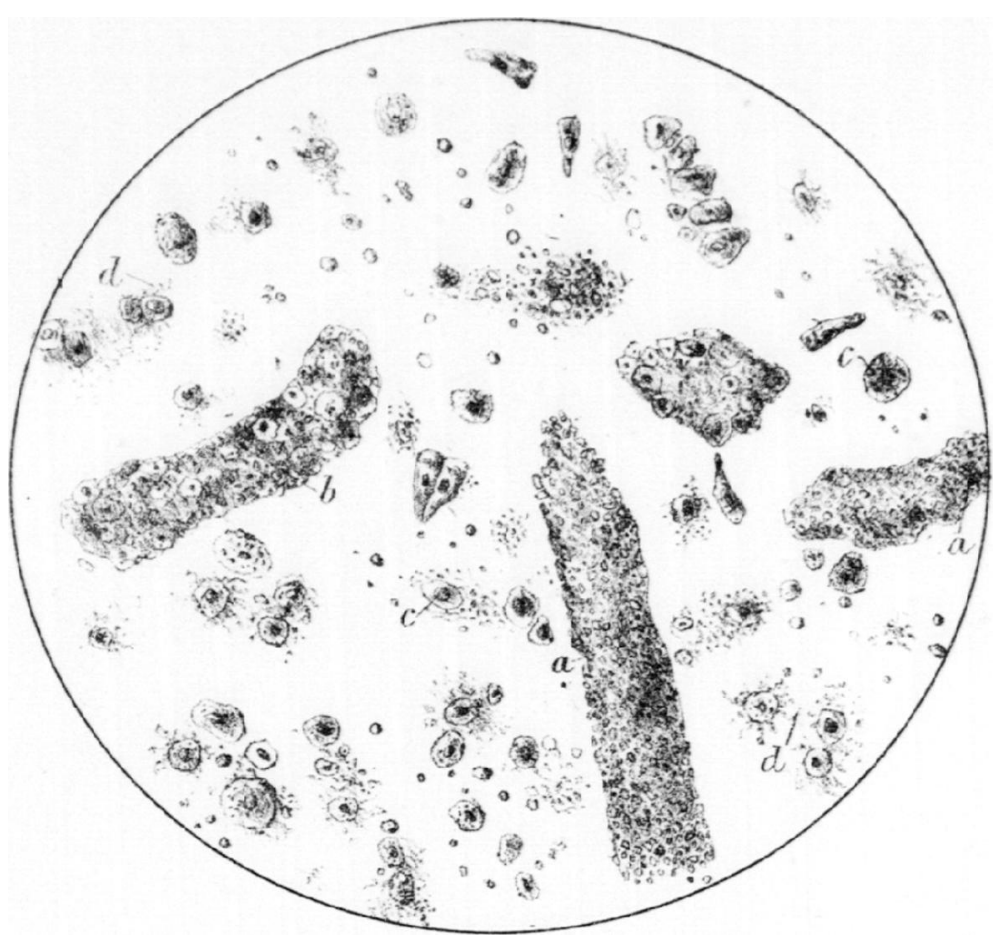

Fig. 2.

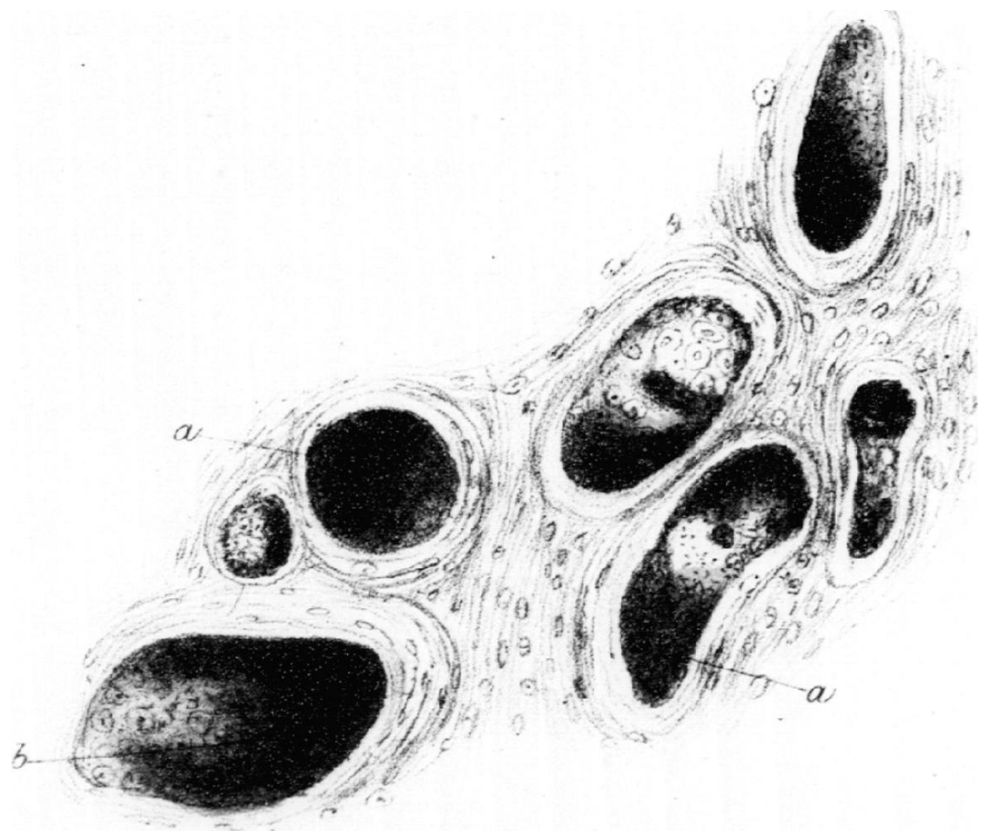

